\title{
Editorial
}

\section{New Achievements in Control of Robotic Systems}

\author{
Víctor Santibáñez, ${ }^{1}$ Arturo Zavala-Río, ${ }^{2}$ Luis Rodolfo García Carrillo, ${ }^{3}$ \\ and Javier Moreno-Valenzuela ${ }^{4}$ \\ ${ }^{1}$ Instituto Tecnológico de La Laguna, 27000 Torreón, COAH, Mexico \\ ${ }^{2}$ Instituto Potosino de Investigación Científica y Tecnológica (IPICYT), 78216 San Luis Potosí, SLP, Mexico \\ ${ }^{3}$ Texas A\&M University-Corpus Christi, Corpus Christi, TX 78412, USA \\ ${ }^{4}$ Instituto Politécnico Nacional, CITEDI, 22510 Tijuana, BC, Mexico
}

Correspondence should be addressed to Víctor Santibáñez; santibanez@ieee.org

Received 27 March 2015; Accepted 27 March 2015

Copyright (C) 2015 Víctor Santibáñez et al. This is an open access article distributed under the Creative Commons Attribution License, which permits unrestricted use, distribution, and reproduction in any medium, provided the original work is properly cited.

\section{Introduction}

Nowadays, robotics is experiencing a noticeable growth mainly due to the successful partnership between theory and practice, whose alliance distinguishes the modern robotics from early robotics. This progress is the result of the interplay between the engineering and scientific communities of different disciplines. In this sense, control engineering plays a major role not only in the development of new robotic systems, but also in the performance improvement of existing and traditional systems. The robot control can be considered as the cornerstone of robotics. Its task consists in driving a robot to accomplish a desired task, in a fully autonomous way. Advanced methods of control have been required in order to adequately face challenging problems which have arisen from underactuation, visual servoing, visual tracking, locomotion, cooperative manipulation, among others.

The aim of this special issue has been to collect novel and original works from the automatic control community in the area of robotic systems, such as industrial robots, legged robots, robot manipulators, cooperative manipulators, and multiagent systems. We hope this collection of papers will provide important and original information to roboticists and control systems researchers. We also consider that the publication of these theoretical, numerical, and experimental contributions will lead to solving some of the challenges that the development of new robotic system demands.

Specifically, this special issue presents the state-of-thescience of several topics of robot control, for example, cooperative tasks and control for mobile robots, pattern generation, intelligent control, and optimization algorithms, as well as new control strategies for fully actuated robots along with their stability analysis.

The papers in this special issue have been organized into three main topics. The first topic is related to intelligent control and optimization algorithms such as the Takagi-Sugeno fuzzy regulators, neural networks, and cooperative evolution algorithms dealing with problems such as the parameter optimization of a snake robot, design of recurrent firstorder neural networks (RFONN) applied to backstepping control of a robot manipulator, and real-time integration of visual servoing and inverse optimal neural control for nonholonomic mobile robots. The second topic takes into account the pattern generation and the control of omnidirectional mobile robots along with multiagents systems. In this domain, control laws to maintain the velocity or to avoid collisions are presented, and some practical problems such as localization of chemicals sources (volatile or toxic chemicals) are analyzed. Finally, the third topic addresses new approaches of control theory along with their stability analysis and applications dealing with modern life challenges in the robotic field. Some of the areas explored by these methods are human-robot interaction tasks, bipedal robot locomotion, and vision systems for the development of strategies for recognition and planning of paths for bipedal locomotion.

A total of thirteen papers have been selected after a fair and rigorous review process. A high rejection rate of $62 \%$ has resulted from the review process, producing a stronger collection of accepted papers in the current special issue. The accepted papers are briefly described below. 


\section{Intelligent Control and Optimization Algorithms}

The paper "Robust Takagi-Sugeno Fuzzy Dynamic Regulator for Trajectory Tracking of a Pendulum-Cart System" by M. A. Llama et al. presents a design of fuzzy controllers for an underactuated system. Starting from a nonlinear model for a pendulum-cart system in which viscous friction is considered, the paper proposes two Takagi-Sugeno fuzzy augmented models, considering in one of them parameter uncertainty. Based on the fuzzy models, three Takagi-Sugeno fuzzy controllers are also proposed via Parallel Distributed Compensation. Real-time experiments consisting of keeping the pendulum in its upright position while the cart follows a reference signal validate the effectiveness of the proposed schemes.

An important growth in the field of evolutive algorithms has occurred recently, where Genetic Algorithm (GA) and Particle Swarm Optimization (PSO) have been used in a great variety of applications with high success. However, both methods have some disadvantages: GA has slow convergence and PSO might be caught in the trap of local optimization. To avoid those inconveniences, approaches that combine these two algorithms have been proposed. In this topic, a novel cooperative evolution algorithm is presented, in the paper "An Optimization Algorithm with Novel RFA-PSO Cooperative Evolution: Applications to Parameter Decision of a Snake Robot" by Q. Gao et al., which is based on a hybrid optimization algorithm with both local and global search capabilities. The method combines the global search property of rain forest algorithm (RFA) and the rapid convergence of PSO to improve the exploration and exploitation property of the agents. Furthermore, an engineering design optimization problem taken from the gait control of a snake-like robot is successfully implemented by the proposed RFA-PSO.

The next paper of this topic is "Real-Time Inverse Optimal Neural Control for Image Based Visual Servoing with Nonholonomic Mobile Robots" by C. López-Franco et al. The main goal of this work is to propose an inverse optimal neural controller for a nonholonomic mobile robot with parameter uncertainties and unknown external disturbances. Here, a discrete-time recurrent high-order neural network (RHONN) is trained with an extended Kalman filter with visual feedback, along with optimal control theory. This method is used to determine the control signals that will force a process to satisfy physical constraints and minimize a performance criterion simultaneously. Commonly, in optimal control theory, a cost functional is defined as function of the state and the control variables; unfortunately, this framework requires solving the Hamilton-Jacobi-Bellman (HTB) equation, which is not an easy task. Taking this into account, C. López-Franco et al. used inverse optimal control to avoid the solution of the HTB equation; indeed, the input of the inverse optimal control is determined by visual feedback.

On the other hand, the wavelet neural networks (WNNs) structure, which has arisen from combining wavelets concepts with artificial neural networks (ANNs) approaches, achieves better identification performance. Furthermore, recurrent wavelet neural networks (RWNNs), which combine properties such as dynamic response of recurrent neural networks (RNNs) and the fast convergence of WNNs, have been used to identify and to control nonlinear systems. The use of WNNs and RWNNs is not a new theme in the literature; however, the implementation of these structures involves the use of multiple-layers with multiple-neurons. Taking this into account, L. A. Vázquez et al. present a novel article entitled "Decentralized Identification and Control in Real-Time of a Robot Manipulator via Recurrent Wavelet First-Order Neural Network." In this paper, a continuoustime decentralized wavelet neural identification and control scheme based on the structure of a recurrent high-order neural networks (RHONN) model with wavelet Morlet activation function is proposed. The resulting structure has been named recurrent wavelet first-order neural network (RWFONN). Furthermore, the design of the proposed controller is carried out using a backstepping approach. The training for the RWFONN is performed online using the filtered error (FE) algorithm and a new neural structure of a single-layer and a single-neuron. The performance of the proposed scheme for trajectory tracking is validated via experimental results performed in a robot manipulator of two DOF with unknown parameters.

\section{Mobile Robots and Multiagents Systems}

The demand for robots with high mobility has increased in the last years. Since the conventional mobile robots do not have high mobility due to their nonholonomic constraints, the omnidirectional mobile robots (OMR) have attracted a lot of attention. These systems have the ability to move simultaneously and independently, in arbitrary directions and orientations in the horizontal plane. Because of its EulerLagrange dynamics, classical control techniques for robot manipulators have been adapted for controlling this kind of mobile robots. An important issue to consider during the control design is the inclusion of the physical limitations of the system, such as bounds in velocities and/or torques. For instance, high velocities developed by the mobile robot can result in a higher probability of wheel slides and deviations from the desired trajectory. Taking this into account, $\mathrm{O}$. Peñaloza-Mejía et al. present the paper "Motion Control Design for an Omnidirectional Mobile Robot Subject to Velocity Constraints," where they focus their attention on the motion control problem of OMR subject to velocity constraints. The proposed control scheme includes explicitly the desired bounds for the developed Cartesian velocities, which in turn bound the maximal velocities developed by the wheels. The proposed method includes a controller to achieve the desired tracking, combined with a passive nonlinear controller which ensures bounded velocities. Also, formal proofs of global asymptotic tracking with desired bounded velocities, along with simulations and experimental results, are provided in this work.

As a consequence of the requirements for an effective controller design, the dynamics of omnidirectional mobile robots have been widely studied. Diverse methodologies have been proposed, for example, models whose simplified geometric relationships determine the robot's position, models 
that take into account the effect of slipping into the dynamical model, and dynamics including motor parameters. This topic is addressed by K. B. Kim et al. in "Finding the Quickest Straight-line Trajectory for a Three-Wheeled Omnidirectional Robot under Input Voltage Constraints." Specifically, they propose an analytical solution to the problem of generating the quickest straight-line trajectory for a three-wheeled omnidirectional mobile robot (TOMR), under the practical constraint of limited voltage, in global coordinates. Additionally, motions with and without rotation of the robot by means of conventional optimization theory are considered.

Another problem encountered in this topic is to trace odor plumes and to locate chemical sources by means of multirobot cooperative strategies. There is a wide range of applications for employing autonomous robots to perform plume tracing and chemical source localization, for example, searching for explosives and demining operations, which can be done by tracing volatile chemicals dispersed from the ordnance. Similar applications include judging toxic or harmful gas leakage location, checking for substance contraband (e.g., heroin), searching for survivors and casualties following a disaster, and antiterrorist attacks. In this topic Y. Zhang et al. propose in "Multiple Chemical Sources Localization Using Virtual Physics-Based Robots with Release Strategy" a novel method for simultaneously locating chemical sources by a virtual physics-based multirobot system. The method makes use of a release strategy, which includes setting a forbidden area, releasing the robots from declared sources, and escaping from it by a rotary force and goal force. This strategy can help to avoid the fact that the robots relocate the same source which has been already located by other robots and leads them to move toward other sources. To validate the proposed methodology, different scenarios were explored simultaneously using a group of six robots or in parallel search by multiple groups of robots.

During the last years, formation control in multiagent systems has received much attention due to the wide range of applications in which they can be used, for example, exploration, search and rescue tasks, and toxic residues cleaning. A very important issue in formation control is the collision avoidance problem, either with other agents or with obstacles. Even if the collision avoidance is ensured, the position among agents can be arbitrarily small, which in real applications where physical dimensions cannot be ignored could imply a collision. The article "Finite-Time Formation Control without Collisions for Multiagent Systems with Communication Graphs Composed of Cyclic Paths" by J. F. Flores-Resendiz et al. proposes a general solution for the case of any number of agents moving on a plane and subjected to a communication graph composed of cyclic paths, which is based on two modifications of the well-known cyclic pursuit algorithm. The control law separately addresses the convergence to the desired formation and the noncollision problems. Furthermore, it ensures that the agents reach the desired geometric pattern in finite time and that they stay at a distance greater than or equal to some prescribed lower bound. Moreover, the closed-loop system does not exhibit undesired equilibria. Numerical simulations and real-time experiments illustrate the good performance of the proposed solution.

\section{Analysis, Control Theory, and Applications}

As it is well known, the linear PID controller is often used in industrial applications for regulation case, because of its simplicity and capability to remove steady-state error caused by external disturbances, uncertainties, and noise. To achieve this functionality, the integrator gain has to be increased; however, under certain circumstances, the stability of the closed-loop system with traditional PID is not guaranteed (from a theoretical point of view). On the other side, when there are external perturbations and system uncertainties, the PID Computed Torque (PID-CT) compensator has been used for the nonlinear trajectory tracking of Lagrangian Nonlinear Dynamical Systems (LNDS). However, the global system convergence of the trivial solution has not been proved. Taking this into account, P. Ordaz et al. propose in this topic a "PID Based on Attractive Ellipsoid Method for Dynamic Uncertain and External Disturbances Rejection in Mechanical Systems," where they deal with the trajectory tracking control problem for LNDS, applied in two degrees of freedom robotic manipulator. A stability analysis is presented, considering the PID-CT for LNDS with unknown but bounded external perturbations, ensuring Uniform-Ultimately Bounded of the solutions.

At present, there exist many control algorithms for regulation task but almost none of them impose a speed behavior; in other words, the main control objective is to reach a desired position without taking into account the velocity. A combination of both goals, that is, to attain a constant state while a constant velocity is kept in the most part of the displacement stage, is, however, a more complicated goal which is analyzed less frequently. In this topic, R. Cuesta et al. propose a control law to face this problem in their article "Robust Tracking and Cruise Control of a Class of Robotic Systems," which is based on a first-order sliding mode with a particular noninvariant, nonconnected surface. The shape of this surface produces indirectly a system behavior which switches from a firstto a second-order sliding mode. First, the control strategy forces the system to attain the noninvariant surface, keeping a constant desired speed while the system moves on the surface. Later, once the system is close to the desired position, it leaves the sliding surface to converge to this position through a second-order sliding mode. The proposed control technique is applied via numerical simulations to a Cartesian robot and experimentally to a rotational mechanism.

In this topic another interesting problem is related to the use of force regulation strategies as fundamental tools to perform tasks with physical interactions among robots, humans, and the environment. In the article "An Optimization-Based Impedance Approach for Robot Force Regulation with Prescribed Force Limits," by R. de J. Portillo-Vélez et al., an optimization-based approach for the regulation of excessive or insufficient forces at the end-effector level is introduced, where the interaction force error at the robot end-effector is minimized, while undesired interaction forces are constrained. The proposal is validated via experimental results for a robotic grasping task.

Among the various motions of a humanoid robot, the most basic and important motion is bipedal walking. In this 
topic, there is also a paper dealing with the legged locomotion case, in which humanoid robots have become quite popular and are used as a research tool worldwide. Neurophysiological studies of animal locomotion have verified that the fundamental rhythmic movements of animals are generated by the central pattern generator (CPG). Q. Lu and J. Tian, in their article "Research on Walking Gait of Biped Robot Based on A Modified CPG Model," have proposed a modified CPG model, whose output function is $\sin (\mathrm{x})$, proving that the modified model has a stable periodic solution and characteristics of rhythmic movement. They show that the modified CPG model can generate spontaneous oscillations, which correspond to biological signals. In order to validate the locomotion model, the authors tested their approach in a sevenlink biped robot where they simulate a cyclic walking gait.

Along the line of developing strategies for locomotion, there exist those where vision systems are used. Currently, most of the vision navigation systems are investigated based on 3-dimensional space. Three-dimensional vision-based navigation needs multicamera or omnidirectional camera systems to provide the robot with data on its orientation and direction. In some cases, the hardware that is needed to implement the algorithm can be more expensive than the robot itself. This fact makes the practical realization of such methods in most of the real-world robotic systems questionable. In the article "Discrete-State-Based Vision Navigation Control Algorithm for One Bipedal Robot" by D. Wei, the author proposed a vision navigation control method based on the socalled by the author "desired direction field." The method uses discrete image sequences to form a discrete state space, which is especially suitable for bipedal walking robots equipped with a single camera and walking on a free-barrier plane surface to track a specific objective. Also, a path planning method is proposed to design the desired direction field and to ensure that the navigation algorithm controls the bipedal robot walking on a plane surface. Simulation results are presented to validate the proposed algorithms.

\section{Acknowledgment}

We thank the reviewers of the journal for the invaluable comments and suggestions given to the authors, which really helped to improve the quality of the papers published in the current special issue.

Víctor Santibáñez Arturo Zavala-Río

Luis Rodolfo García Carrillo Javier Moreno-Valenzuela 


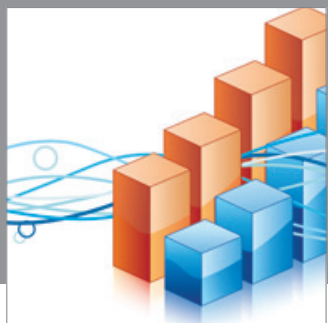

Advances in

Operations Research

mansans

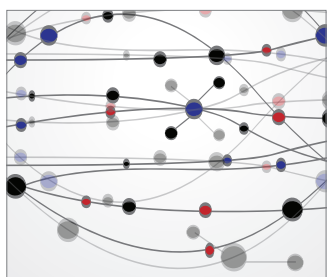

The Scientific World Journal
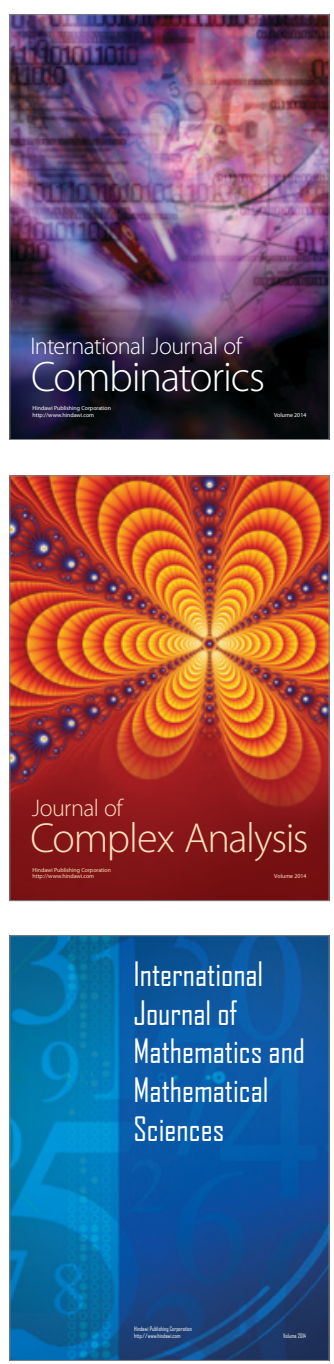
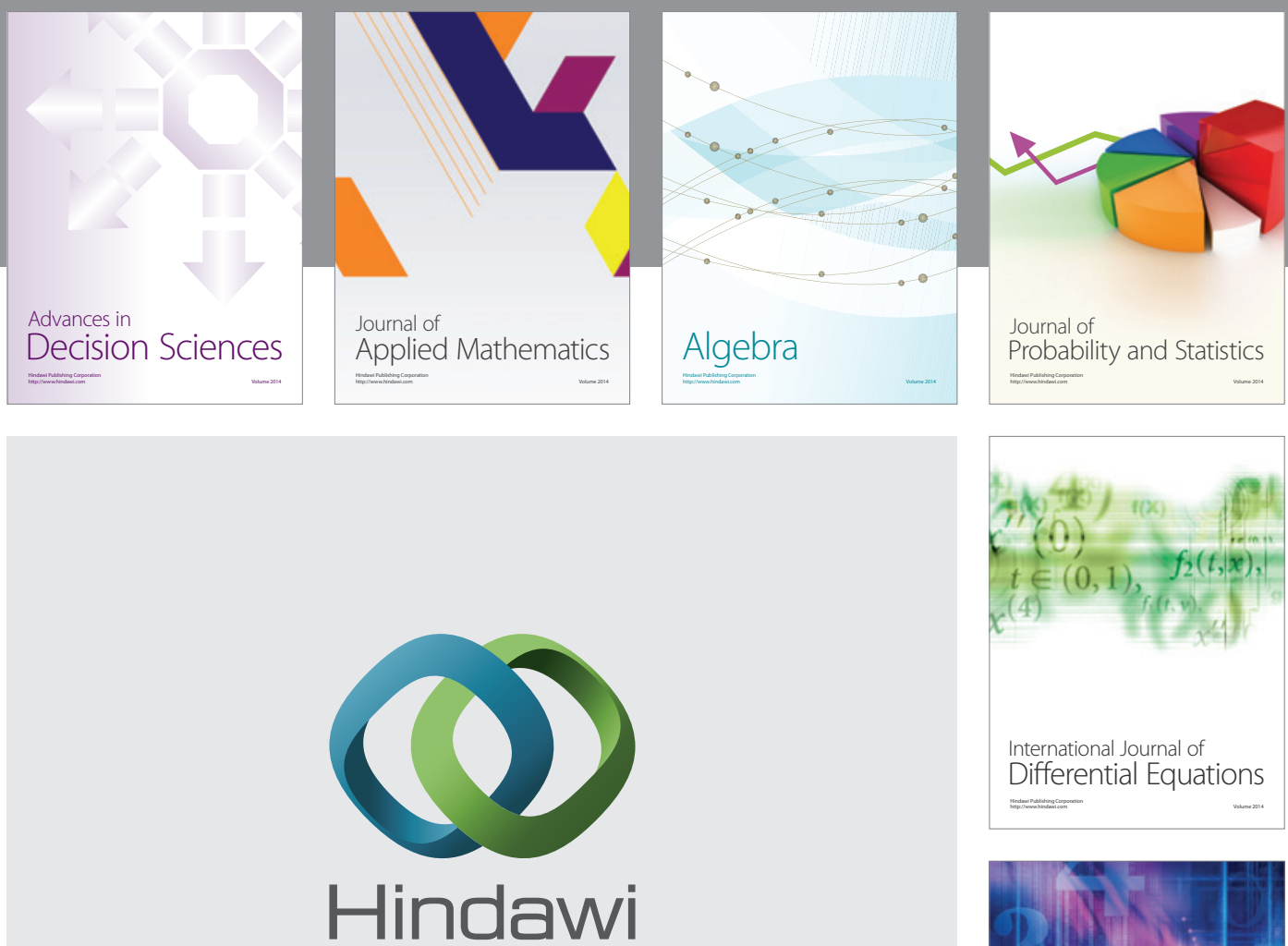

Submit your manuscripts at http://www.hindawi.com
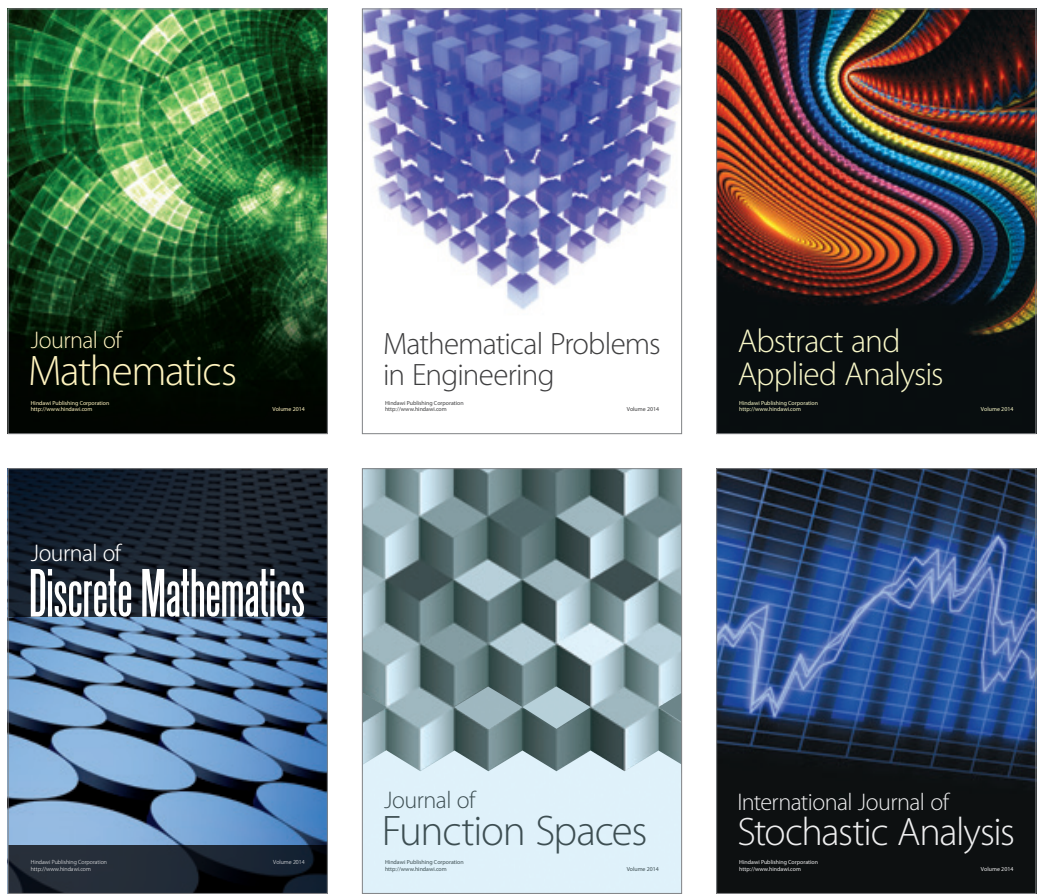

Journal of

Function Spaces

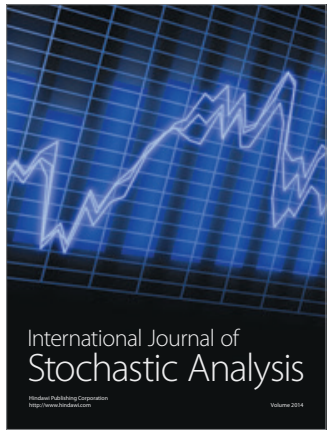

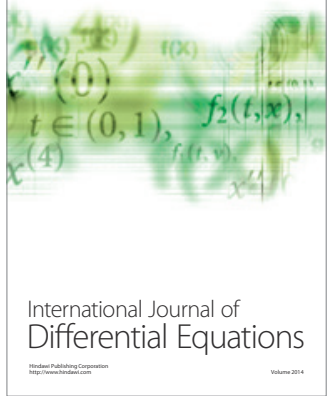
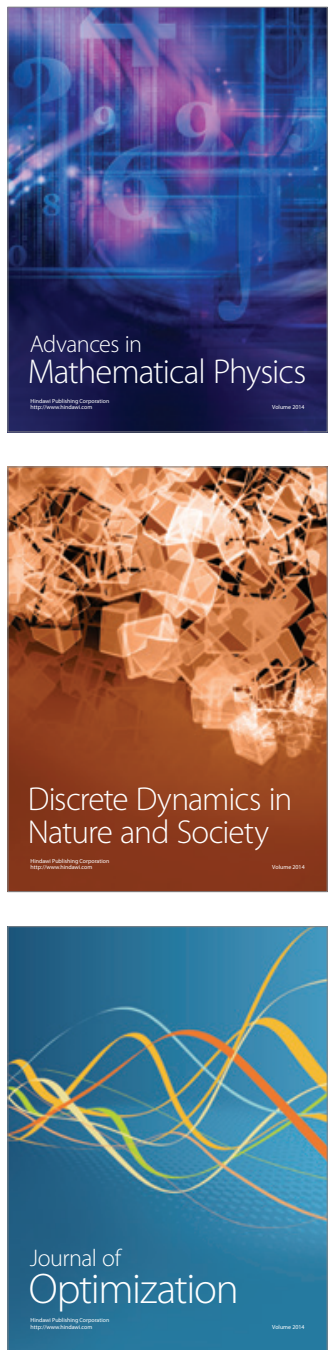